\title{
The effect of the NMDA receptor antagonist MK-801 on the acquisition and extinction of learned fear in the developing rat
}

\author{
Julia M. Langton, ${ }^{1}$ Jee Hyun Kim, Jennifer Nicholas, and Rick Richardson \\ School of Psychology, The University of New South Wales, Sydney 2052, Australia
}

\begin{abstract}
Recent findings reveal qualitative developmental differences in extinction of learned fear. The present study explored potential developmental differences in the role of NMDA in acquisition and extinction. Rats were injected with MK-801 prior to fear conditioning or extinction training. Acquisition was found to be NMDA dependent in both age groups, whereas extinction was found to be NMDA dependent in 23-day-old rats, but NMDA independent in 16-day-old rats. These results illustrate another fundamental developmental difference in extinction as well as a dissociation in the role of NMDA in the acquisition and extinction of fear early in development.
\end{abstract}

The acquisition of fear is commonly studied with Pavlovian conditioning procedures in which a neutral conditioned stimulus (CS; e.g., tone) is paired with an aversive unconditioned stimulus (US; e.g., footshock). Subsequent presentations of the CS elicit a number of fear responses (e.g., freezing), but these reactions can be extinguished by repeatedly presenting the CS in the absence of the US. Previous research shows that both the acquisition and extinction of learned fear involves N-methyl-D-aspartate (NMDA) receptors. Specifically, infusing APV (a selective NMDA antagonist) into the amygdala impairs acquisition of fear conditioning (Miserendino et al. 1990) and extinction (Falls et al. 1992). Baker and Azorlosa (1996) also found that NMDA antagonists impair long-term extinction by giving systemic injections of MK-801.

While NMDA is involved in both the acquisition and extinction of learned fear in adult rats, the role of NMDA in these processes has not been systematically examined during development. Further, recent studies suggest that the mechanisms underlying extinction are qualitatively different during development. More specifically, renewal and reinstatement are observed in adult rats (see Bouton 2002) and postnatal day (PND) 23 rats; however, neither occur in PND 16 rats (Kim and Richardson 2007a,b; Yap and Richardson 2007). Likewise, pre-test administration of the GABA inverse agonist FG-7142 results in a return of conditioned responding after extinction training in PND 23 (Kim and Richardson 2007a) and adult rats (Harris and Westbrook 1998), but not in PND 16 rats. Taken together, these findings provide support for the idea that the processes mediating extinction are not uniform across development.

This study explored developmental differences in the role of NMDA in the acquisition and extinction of learned fear. Specifically, the effect of the NMDA receptor antagonist MK-801 on fear conditioning and extinction was examined in PND 16 and PND 23 rats. In each experiment, the group label refers to the age at which fear conditioning occurred.

The fear conditioning and extinction procedures used in the current study were the same as previous studies from our laboratory (see Kim and Richardson 2007a for details). MK-801 (Sigma-Aldrich) or saline was administered subcutaneously at a volume of $2 \mathrm{~mL} / \mathrm{kg}$.

\section{1'Corresponding author.}

E-mail jlangton@psy.unsw.edu.au; fax 61-2-93853641.

Article is online at http://www.learnmem.org/cgi/doi/10.1101//m.692407.
First, we examined whether there are age-related differences in the role of NMDA in Pavlovian fear conditioning. Specifically, PND 17 and PND 24 rats were injected with MK-801 $(0.1 \mathrm{mg} / \mathrm{kg})$ or saline $10 \mathrm{~min}$ before fear conditioning to an auditory CS and tested for CS-elicited fear the following day.

At both ages, rats injected with saline before fear conditioning showed higher levels of freezing at test than did rats injected with MK-801 (Fig. 1). A two-way ANOVA revealed a significant effect of drug, $F_{(1,37)}=15.77, P<0.001$, but no effect of age, $F_{(1,37)}=1.04, P=0.31$, and no significant age-by-drug interaction, $F<1.0$.

We also examined whether there are age-related differences in the role of NMDA in the extinction of learned fear. PND 16 and PND 23 rats were trained to fear an auditory CS. The following day rats received an injection of MK-801 $(0.1 \mathrm{mg} / \mathrm{kg})$ or saline 10 min before extinction training. A no-extinction control group was injected with saline and placed in the extinction context for the same period of time, but not exposed to the CS. The following day, all animals were tested for CS-elicited fear.

Regardless of age or drug administered before the extinction session, all rats showed within-session extinction (Fig. 2A). That is, rats displayed high levels of freezing during the first 2 min of extinction training that decreased by the last 2 min of extinction training. A three-way ANOVA revealed a significant effect of block, $F_{(1,36)}=33.90, P<0.001$, but no effect of age, $F<1.0$, drug, $F_{(1,36)}=1.77, P=0.19$, or age-by-drug interaction, $F<1.0$.

Although there were no age- or drug-related differences in within-session extinction, there were differences the following day when retention of the extinction memory was assessed (Fig. 2B). Specifically, both PND 16 and PND 23 rats in the saline condition retained the low levels of freezing seen at the end of extinction training. PND 16 rats in the MK-801 condition exhibited levels of freezing comparable to that seen in the saline controls, but PND 23 rats in the MK-801 condition exhibited substantially higher levels of freezing than did their saline controls. Therefore, while administration of MK-801 prior to extinction training had no effect on within-session extinction at either age, it impaired the retention of the extinction memory in PND 23 rats but had no effect on long-term extinction in PND 16 rats. Moreover, both PND 16 and PND 23 rats in the no-extinction control condition exhibited higher levels of freezing than extinguished rats given saline in both age groups and PND 16 rats in the MK-801 condition. The performance of these nonextin- 


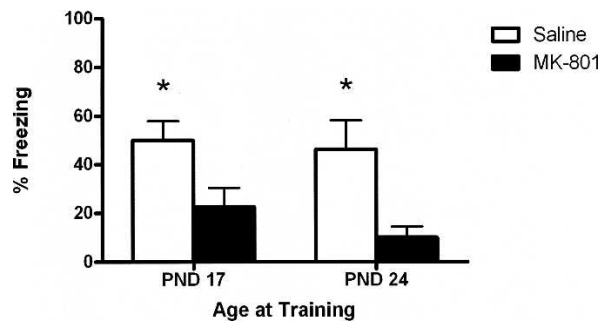

Figure 1. Acquisition of learned fear is NMDA dependent in both PND 17 and PND 24 rats. Mean ( \pm SEM) freezing in response to the CS during test. Rats of two ages (postnatal day 17 and 24) were in one of two conditions: PND 17-saline $(n=12)$, PND 17-MK-801 $(n=11)$, PND $24-$ saline $(n=9)$, PND 24-MK-801 $(n=9)$. ( $\left.{ }^{*}\right)$ A significant difference from the other group of the same age.

guished controls shows that rats at each age did not merely forget the original CS-US association over time.

Statistical analyses confirmed these descriptions of the results. A two-way ANOVA revealed significant effects of age, $F_{(1,36)}=19.12, P<0.001$, and group, $F_{(1,36)}=24.14, P<0.001$, as well as a significant age-by-group interaction, $F_{(1,36)}=7.18$, $P<0.05$. Post-hoc comparisons with Tukey's HSD procedure showed that this interaction was due to a significant difference between PND 16 and PND 23 rats in the MK-801 condition, $(P<0.001)$, whereas PND 16 and PND 23 rats did not differ in the saline or no-extinction conditions.

In order to ensure that this difference was not due to a developmental difference in the dose response function, we examined the effect of different doses of MK-801 $(0.05,0.1$, or 0.2 $\mathrm{mg} / \mathrm{kg}$ ) on extinction in PND 16 rats. Regardless of dose, all rats showed within-session extinction (Fig. 3A). A mixed-model ANOVA revealed a significant effect of block, $F_{(1,35)}=124.11$, $P<0.001$. At test the following day, the no-extinction control group exhibited higher levels of freezing compared with all of the extinguished rats, regardless of drug dose (Fig. 3B). This description of the results was confirmed by statistical analysis. ANOVA revealed a significant effect of group, $F_{(4,46)}=5.23, P<0.05$. Posthoc comparisons using Tukey's HSD procedure showed that this difference was due to a significant difference between the noextinction group and all of the other groups $(P<0.05)$. There were no significant differences between any of the other groups.

Although NMDA is required for the acquisition and extinction of learned fear in adult rats, until the present experiments, the involvement of NMDA in these processes had not been examined across development. While a pre-training injection of MK-801 impaired retention of acquisition at both ages, a preextinction injection of MK-801 attenuated retention of extinction only in PND 23 rats. The finding that MK-801 impaired acquisition in both age groups demonstrates that, like in adult rats, acquisition is NMDA dependent in the developing rat as well. However, the finding that MK-801 only impaired retention of extinction in PND 23 rats suggests that unlike adult rats, extinction is NMDA independent in young rats (PND 16). These latter results converge with a number of other recent findings that show qualitative developmental differences in the extinction of learned fear (Kim and Richardson 2007a,b; Yap and Richardson 2007).

These results have several important implications for understanding extinction. Although extinction is procedurally simple, it is not completely understood at either the theoretical or neural level. The view that extinction is a type of new learning rather than a type of unlearning or erasure is supported by phenomena such as renewal, reinstatement, and spontaneous recovery. In each of these phenomena a learned fear response returns following extinction, even though no additional CS-US pairings occur, thereby showing that the original CS-US memory remains intact after extinction training. Moreover, studies showing that blocking NMDA receptor activation impairs extinction also suggest that extinction is a type of new learning that is vulnerable to disruption by the same manipulations that affect the acquisition of learned fear. However, because recovery of extinguished fear (e.g., renewal) is seldom complete (see Delamater 2004), it is clear that one theory is not adequate to explain extinction and perhaps unlearning and new learning as well as nonassociative processes are involved (see Myers and Davis 2007). Therefore, it seems that a hybrid model that incorporates numerous processes is more appropriate to explain extinction.

It has been suggested that whatever processes are recruited during extinction may depend on parameters such as the timing of extinction training (Myers and Davis 2007). For example, if extinction training occurs immediately after acquisition, then unlearning processes may be dominant, whereas if extinction training occurs $72 \mathrm{~h}$ after acquisition, then new learning may be the dominant process. In support of this, Myers et al. (2006) observe attenuated renewal, reinstatement, and spontaneous recovery in rats extinguished immediately after fear conditioning, results that mirror those seen in PND 16 rats following extinction training. Although extinction training occurred $24 \mathrm{~h}$ after fear conditioning in PND 16 rats, the failure to observe renewal, reinstatement, return of conditioned responding with FG-7142 (Kim and Richardson 2007a,b), or attenuation of extinction with MK-801 all suggest that extinction may be unlearning in PND 16 rats. In other words, all of the manipulations that provide evidence for the new-learning account of extinction are not observed in PND 16 rats. Therefore, extinction in young rats (PND 16 ) is an instance where the dominant process during extinction is not new learning.

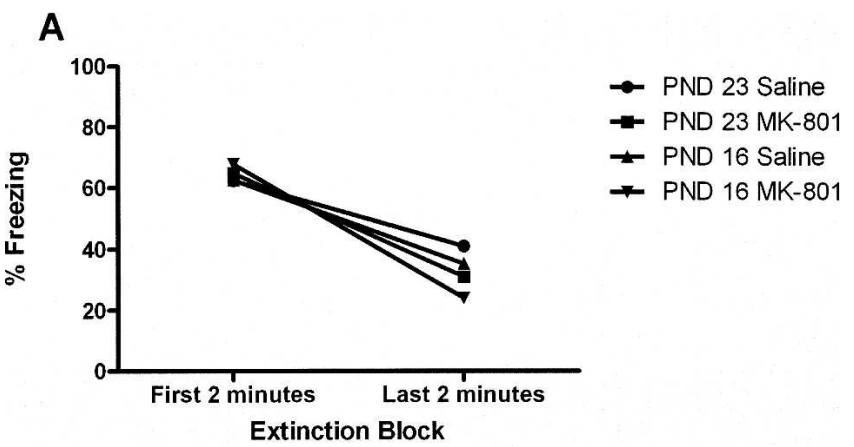

$$
\text { B }
$$

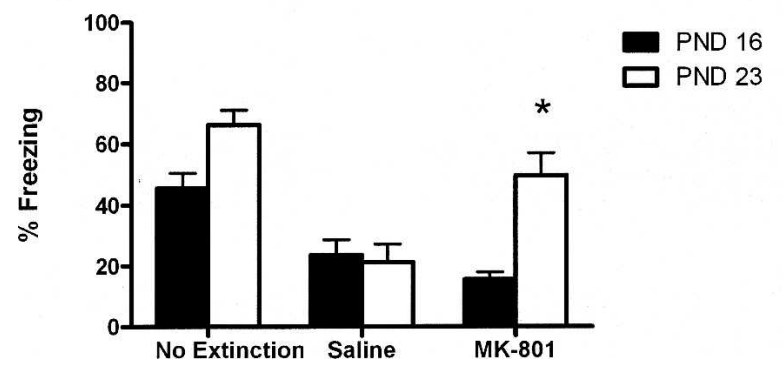

Figure 2. Retention of extinction is NMDA dependent in PND 23 but not PND 16 rats. $(A)$ Mean freezing of rats in response to the auditory CS during the first $2 \mathrm{~min}$ and last $2 \mathrm{~min}$ of extinction training. (B) Mean $( \pm$ SEM) freezing in response to the CS during test. Rats of two ages (postnatal day 16 and 23) were in one of three conditions: PND 16, no extinction $(n=11)$; PND 16-saline $(n=13)$; PND 16-MK-801 $(n=11)$; PND 23-no extinction $(n=8)$; PND 23-saline $(n=8)$; PND 23-MK-801 $(n=8) .\left({ }^{*}\right)$ A significant difference from the other age group in the same condition. 


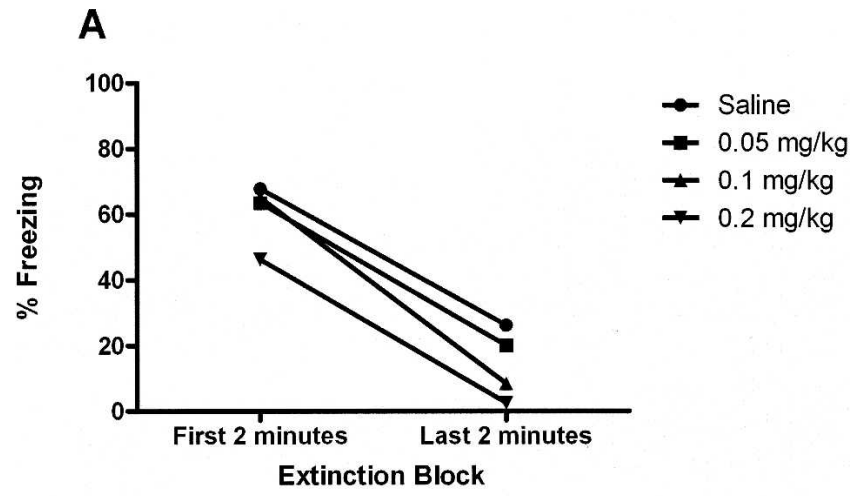

B

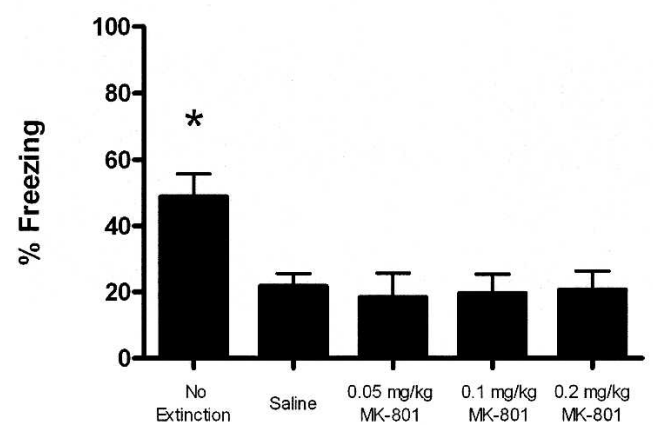

Figure 3. Retention of extinction is NMDA-independent in PND 16 rats regardless of the dose of MK-801 administered. (A) Mean freezing of rats in response to the auditory CS during the first $2 \mathrm{~min}$ and last $2 \mathrm{~min}$ of extinction training. (B) Mean ( \pm SEM) freezing in response to the CS during test the day following extinction training. Postnatal day 16 rats were in one of five conditions: no extinction $(n=12)$; saline $(n=12)$; MK-801 $0.05 \mathrm{mg} / \mathrm{kg}(n=9) ;$ MK-801 $0.1 \mathrm{mg} / \mathrm{kg}(n=10) ;$ MK-801 0.2 $\mathrm{mg} / \mathrm{kg}(n=10)$. ( $\left.{ }^{*}\right)$ A significant difference from the other groups.

An alternative explanation for the observed age-related differences in the effect of NMDA receptor blockade on extinction could be that there are developmental differences in the neural circuitry involved in extinction. The current neural model of extinction involves interactions between the amygdala, hippocampus, and medial prefrontal cortex (PFC) (Sotres-Bayon et al. 2004). These structures, or the connections between them, may not be fully developed in PND 16 rats. Although it is clear that NMDA receptors are fully functioning in the amygdala at least in regard to the acquisition of learned fear in PND 16 rats, NMDA receptors may not be fully functioning throughout the neural circuitry involved in extinction. For instance, it is well documented that PND 16 rats are impaired at hippocampally mediated tasks such as contextual fear conditioning, which has led to suggestions that PND16 rats have an underdeveloped hippocampus (Rudy 1993). An underdeveloped hippocampus in PND 16 rats may explain the lack of a contextually bound extinction memory in these rats. Likewise, there is evidence that the cortical thickness of the PFC is not adult-like at PND 14-18, but reaches adult thickness by PND 24 (Van Eden and Uylings 1985). Further, infusion of the NMDA antagonist CPP into the PFC prior to or shortly after extinction training blocks extinction but not acquisition of learned fear in adult rats (Burgos-Robles et al. 2007), which suggests that NMDA receptors in the PFC are required for the consolidation of an inhibitory extinction memory. Therefore, future studies should examine whether there are age-related differences in the neural circuitry mediating extinction. Additionally, findings that within-session extinction is both NMDA independent (Baker and Azorlosa 1996; Santini et al. 2001; but see Sotres-Bayon et al. 2007) and PFC independent (Quirk et al. 2000) can account for the result that PND 16 rats show withinsession extinction comparable to PND 23 rats and differ only in the mechanisms involved in long-term extinction.

As well as showing developmental differences in the role of NMDA receptors in extinction, the current findings also show a dissociation in the role of NMDA receptors in the acquisition and extinction of learned fear in PND 16 rats. Specifically, PND 16 rats are extremely well-equipped to extinguish fear memories and unlike acquisition, this process is not affected by blocking NMDA receptors. It is well-documented that the translation of shortterm to long-term memory relies on activation of a complex intracellular signaling cascade involving NMDA receptors (Kandel 2001). However, the current results show that this process is involved in acquisition but not extinction in PND 16 rats. Therefore, some other process must be responsible for the translation from short-term to long-term extinction memory in PND 16 rats. In adult rats, multiple neurotransmitter systems (e.g., NMDA, GABA, and opiates) are involved in extinction. However, as neither NMDA or GABA seem to be involved in extinction in PND 16 rats (present results; Kim and Richardson 2007a), one potential mechanism for extinction in young rats is the endogenous opioid system. This neurotransmitter system has been found to regulate fear conditioning (McNally and Westbrook 2003) and extinction (McNally et al. 2004) in adult rats. Moreover, the opioid system is a likely mechanism for mediating extinction in young rats, as it is active from birth (Blass et al. 1991) and has been found to modulate memory in infant rats (Weber et al. 2006).

Finally, the converging evidence showing that extinction in PND 16 rats is qualitatively different from extinction in PND 23 and adult rats may have implications for understanding the nature of anxiety disorders in childhood. It has been proposed that early experiences have extensive effects on later behavior (Mineka and Zinbarg 2006), and longitudinal data suggest that anxiety disorders are the most prevalent disorders in childhood and if left untreated, persist into adulthood (Newman et al. 1996). While there is no doubt that exposure-based therapies used to treat anxiety disorders are very successful, relapse is a common problem in adult populations (Rachman 1989). However, results demonstrating that extinction in PND 16 rats is not disrupted by an NMDA antagonist and that PND 16 rats do not demonstrate renewal or reinstatement (Kim and Richardson $2007 a, b)$ seem to suggest that exposure therapy in young children may make them less susceptible to relapse compared with exposure therapy in adults. Further, in contrast to suggestions that fear acquired early in development has enduring effects that are resistant to extinction (Jacobs and Nadel 1985, 1999), our recent preclinical studies on the developmental differences in extinction suggest that fear acquired early in development may be more readily and more permanently extinguished than fear acquired later in development.

In conclusion, the results of this study provide insight into the mechanisms involved in the acquisition and extinction of learned fear. Importantly, the results in PND 23 rats converge with findings in adult rats that implicate NMDA receptors in the acquisition and extinction of learned fear. However, the developmental differences in the role of NMDA in extinction contribute vital information to a seemingly simple, yet increasingly complex phenomenon. In other words, the results in PND 16 rats provide an instance when extinction is NMDA independent. This is a novel finding and one that should be taken into account in future examinations of the role of different neurotransmitter systems in extinction. Additionally, the results represent another example where extinction may be dominated by unlearning processes rather than new learning processes (Myers et al. 2006). 
Finally, these findings contribute to the growing body of evidence illustrating qualitative differences in the processes involved in extinction across development (Kim and Richardson 2007a,b; Yap and Richardson 2007).

\section{Acknowledgments}

This research was supported by an Australian Postgraduate Award (J.M.L. and J.H.K.) and Australian Research Council grant DP0666953 (R.R.).

\section{References}

Baker, J.D. and Azorlosa, J.L. 1996. The NMDA antagonist MK-801 blocks the extinction of Pavlovian fear conditioning. Behav. Neurosci. 110: $618-620$

Blass, E.M., Jackson, A.M., and Smotherman, W.P. 1991. Milk-induced opioid-mediated antinociception in rats at the time of cesarean delivery. Behav. Neurosci. 105: 677-686.

Bouton, M.E. 2002. Context, ambiguity, and unlearning: Sources of relapse after behavioral extinction. Biol. Psychiatry 52: 976-986.

Burgos-Robles, A., Vidal-Gonzalez, I., Santini, E., and Quirk, G.J. 2007. Consolidation of fear extinction requires NMDA receptor-dependent bursting in the ventromedial prefrontal cortex. Neuron 53: 871-880.

Delamater, A.R. 2004. Experimental extinction in Pavlovian conditioning: Behavioural and neuroscience perspectives. Q. J. Exp. Psychol. B 57: 97-132.

Falls, W.A., Miserendino, M.J., and Davis, M. 1992. Extinction of fear-potentiated startle: Blockade by infusion of an NMDA antagonist into the amygdala. J. Neurosci. 12: 854-863.

Harris, J.A. and Westbrook, R.F. 1998. Evidence that GABA transmission mediates context-specific extinction of learned fear. Psychopharmacology 140: 105-115.

Jacobs, W.J. and Nadel, L. 1985. Stress-induced recovery of fears and phobias. Psychol. Rev. 92: 512-531.

Jacobs, W.J. and Nadel, L. 1999. The first panic attack: A neurobiological theory. Can. J. Exp. Psychol. 53: 92-107.

Kandel, E.R. 2001. The molecular biology of memory storage: A dialogue between genes and synapses. Science 294: 1030-1038.

Kim, J.H. and Richardson, R. 2007a. A developmental dissociation of context and GABA effects on extinguished fear in rats. Behav. Neurosci. 121: 131-139.

Kim, J.H. and Richardson, R. 2007b. A developmental dissociation in reinstatement of an extinguished fear response in rats. Neurobiol. Learn. Mem. 88: 48-57.

McNally, G.P. and Westbrook, R.F. 2003. Temporally graded, context-specific retrograde amnesia and its alleviation by context preexposure: Effects of postconditioning exposures to morphine in the rat. J. Exp. Psychol. Anim. Behav. Process. 29: 130-142.

McNally, G.P., Pigg, M., and Weidemann, G. 2004. Opioid receptors in the midbrain periaqueductal gray regulate extinction of Pavlovian fear conditioning. J. Neurosci. 24: 6912-6919.

Mineka, S. and Zinbarg, R. 2006. A contemporary learning theory perspective on the etiology of anxiety disorders. Am. Psychol. 61: 10-26.

Miserendino, M.J., Sananes, C.B., Melia, K.R., and Davis, M. 1990. Blocking of acquisition but not expression of conditioned fear-potentiated startle by NMDA antagonists in the amygdala. Nature 345: 716-718.

Myers, K.M. and Davis, M. 2007. Mechanisms of fear extinction. Mol. Psychiatry 12: 120-150.

Myers, K.M., Ressler, K.J., and Davis, M. 2006. Different mechanisms of fear extinction dependent on length of time since fear acquisition. Learn. Mem. 13: 216-223.

Newman, D.M., Moffit, T.E., Caspi, A., Magdol, L., Silva, P.A., and Stanton, W.R. 1996. Psychiatric disorder in a birth cohort of young adults: Prevalence, comorbidity, clinical significance, and new case incidence from ages 11 to 21. J. Consult. Clin. Psychol. 64: 552-562.

Quirk, G.J., Russo, G.K., Baron, J.L., and Lebron, K. 2000. The role of ventromedial prefrontal cortex in the recovery of extinguished fear. J. Neurosci. 20: 6225-6231.

Rachman, S. 1989. The return of fear: Review and prospect. Clin. Psychol. Rev. 9: 147-168.

Rudy, J.W. 1993. Contextual conditioning and auditory cue conditioning dissociate during development. Behav. Neurosci. 107: $887-891$.

Santini, E., Muller, R.U., and Quirk, G.J. 2001. Consolidation of extinction learning involves transfer from NMDA-independent to NMDA-dependent memory. I. Neurosci. 21: 9009-9017.

Sotres-Bayon, F., Bush, D.E., and LeDoux, J.E. 2004. Emotional perseveration: An update on prefrontal-amygdala interactions in fear extinction. Learn. Mem. 11: 525-535.

Sotres-Bayon, F., Bush, D.E., and LeDoux, J.E. 2007. Acquisition of fear extinction requires activation of the NR2B-containing NMDA receptors in the lateral amygdala. Neuropsychopharmacology 1: 1-12.

Van Eden, C.G. and Uylings, B.H. 1985. Cytoarchitectonic development of the prefrontal cortex in the rat. J. Comp. Neurol. 241: 253-267.

Weber, M., McNally, G.P., and Richardson, R. 2006. Opioid receptors regulate retrieval of infant fear memories: Effects of naloxone on infantile amnesia. Behav. Neurosci. 120: 702-709.

Yap, C.S.L. and Richardson, R. 2007. Extinction in the developing rat: An examination of renewal effects. Dev. Psychobiol. 49: 565-575.

Received July 2, 2007; accepted in revised form August 3, 2007. 


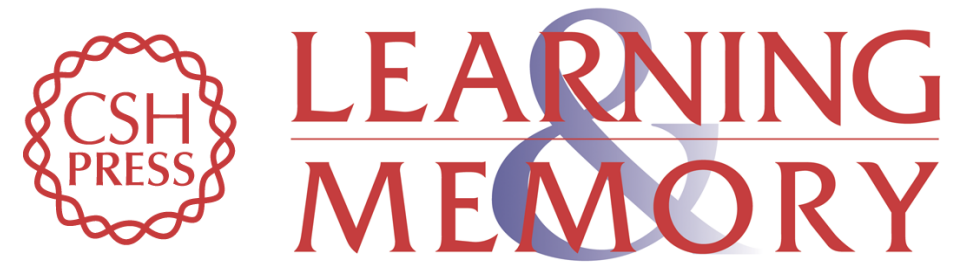

\section{The effect of the NMDA receptor antagonist MK-801 on the acquisition and extinction of learned fear in the developing rat}

Julia M. Langton, Jee Hyun Kim, Jennifer Nicholas, et al.

Learn. Mem. 2007, 14:

Access the most recent version at doi:10.1101//m.692407

References This article cites 28 articles, 7 of which can be accessed free at:

http://learnmem.cshlp.org/content/14/10/665.full.html\#ref-list-1

License

Email Alerting Receive free email alerts when new articles cite this article - sign up in the box at the Service top right corner of the article or click here. 